Editorial

\title{
Do We Need Early ACPA Testing in Inflammatory Arthritis?
}

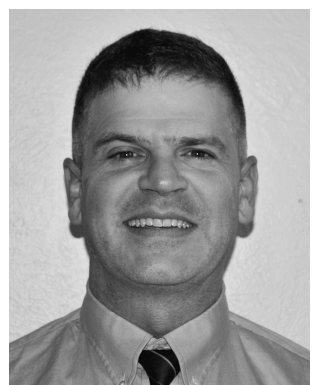

Testing is an integral part of the diagnosis and management of patients with rheumatic disease. However, in this era of increased focus on healthcare costs, it is important to understand exactly how tests affect the delivery and costs of care. Along these lines, the American Board of Internal Medicine has begun the Choosing Wisely program with the goal "of advancing a national dialogue on avoiding wasteful or unnecessary medical tests, treatments and procedures" ${ }^{\text {. Multiple }}$ agencies in the United States and Canada are participating in this campaign, including the American College of Rheumatology (ACR), which has created a set "Five Things that Physicians and Patients Should Consider" in rheumatology-related testing ${ }^{2}$. These are important efforts, which hopefully will ultimately lead to improved clinical care, as well as improved costs of care, based on sound scientific investigations.

Two tests that are used often in the evaluation of inflammatory arthritis (IA) are rheumatoid factor (RF) and anticitrullinated protein antibodies (ACPA), with both of these tests being included in the 2010 ACR/European League Against Rheumatism (EULAR) classification criteria for rheumatoid arthritis (RA) ${ }^{3}$. Notably, these autoantibody tests are not included in the Choosing Wisely campaign. However, in the Canadian healthcare system, ACPA testing is not uniformly paid for, and as such a subset of patients with IA and RA do not have this testing performed. This has led to an opportunity for Shu and colleagues, whose report is published in the November 2015 issue of The Journal ${ }^{4}$, to catch the spirit of the Choosing Wisely campaign and to explore the relationship between ACPA testing and the short-term management of patients with early IA.

Using data from the Canadian early ArThritis CoHort (CATCH), the authors identified a total of 1998 patients seen since 2007 in rheumatology clinics with early IA defined as persistent synovitis for more than 6 weeks but less than 12 months, and then followed by trained rheumatologists. For these analyses, these patients were divided into 3 groups based on autoantibody testing: Group 1 was RF-positive and/or ACPA-positive ( $\mathrm{n}=1276)$; Group 2 was RF-negative and ACPA-negative ( $\mathrm{n}=497)$; and Group 3 was RF-negative and their ACPA status unknown because testing was not performed $(n=225)$. The patients were followed from baseline to 3 months, and differences between their disease classification status, treatments, and disease activity were evaluated.

The authors found that more patients in Group 1 fulfilled the 2010 ACR/EULAR criteria for RA. In addition, patients in Groups 1 and 2 were receiving more disease-modifying antirheumatic drugs (DMARD) at 3 months than those in Group 3, although corticosteroid use was similar across all groups. However, at 3 months, patients in Group 3 had similar overall disease activity as Groups 1 and 2 , as measured by the Disease Activity Score (DAS) and Health Assessment Questionnaire (HAQ). No direct cost analyses were performed, and no results from any additional testing for ACPA in Group 3 were reported.

To explain the finding that the ACPA-untested group was taking fewer DMARD than the other groups despite similar levels of disease activity as measured by the DAS and HAQ, the authors made the point that perhaps lack of ACPA testing influenced early treatment decisions specifically because of decreased confidence in the clinical diagnosis and prognosis. However, the authors also concluded that if these results are confirmed in other, longer-term studies that "perhaps in [early IA] ACPA does not add value with respect to early outcomes."

With the Choosing Wisely concepts of avoiding "wasteful or unnecessary tests" as background, this type of study that seeks to identify the utility of testing in the management of rheumatic disease is welcome. However, there are yet many unanswered questions before the field can arrive at robust guidelines for the optimal use of testing in general in rheumatic disease, and in particular the use of RF and ACPA in the early management of IA. In particular, we need to know the specific cost differences between RF and ACPA testing in a variety of clinical models. What if ACPA were equal in cost or even less expensive than RF? Would it still make sense to avoid ACPA testing in the early evaluation of IA? In particular, there have been several studies, including

See ACPA and short-term outcomes in early arthritis, J Rheumatol 2015;42(11):2023

Personal non-commercial use only. The Journal of Rheumatology Copyright (C) 2016. All rights reserved. 
a large systematic review by Whiting and colleagues ${ }^{5,6}$ suggesting that, given their higher specificity, ACPA tests may be preferable to RF. Also, how do the costs of early RF and ACPA testing affect longterm outcomes, especially beyond the relatively short 3-month interval evaluated by Shu and colleagues? Further, because a growing body of data supports that one of the best ways to improve outcomes in IA and RA is to identify and treat patients with disease earlier ${ }^{7}$, could limiting ACPA testing delay referrals to rheumatologists? Moreover, as Shu and colleagues point out, because early ACPA positivity could influence confidence in using DMARD therapy early, if in studies with longer-term followup, the earlier use of DMARD in patients with ACPA testing was associated with improved longterm outcomes (as well as perhaps reduced use of more potentially toxic medications such as corticosteroids), would reduced testing be of benefit?

Additionally, while Shu and colleagues did not investigate this specific issue, it is of high interest to know how many subjects with IA or RA who were RF-negative would be ACPA-positive, and how that knowledge would affect clinicians' management. Work by Lee and Schur identified that 10 of 29 RF-negative patients (34\%) with RA were ACPA-positive ${ }^{6}$. That rate may not be found in all patients; however, if it was found that ACPA positivity improved referral patterns, drove earlier use of DMARD, and improved outcomes overall, ACPA testing could lead to substantial longterm benefit even if ACPA were present in only a small percentage of RF-negative patients. In addition, given that there are some data to indicate that drugs such as rituximab (RTX) may be more effective in seropositive $\mathrm{RA}^{8}$, would broader understanding of RF and ACPA positivity in early IA help guide future therapy?

An issue underlies many of these questions: What is the minimal amount of testing needed to initially evaluate and manage IA? Or, more dramatically, could IA initially be managed based on patients' symptoms and examination findings alone, with more advanced testing done only when additional treatment decisions are necessary? For example, if a patient with RA (by historical and clinical examination criteria) fails to respond to initial methotrexate (MTX), the physician would then order RF and ACPA testing; and if test results were positive, determine whether RTX is a reasonable next step. However, this type of approach is unlikely to work well because a broad range of diseases can present with IA, and most rheumatologists would agree that early testing is necessary to understand the extent of disease and tissue injury. For example, one would not want to treat someone with IA for an RA-like disease with MTX or low-dose steroids if the real issue was systemic lupus erythematosus and the patient also had nephritis.

Also, while the history and physical examination are likely the best first "tests" to identify IA, how good are clinicians in the identification of IA where even expert rheumatologists may disagree on its presence (or absence)? Could we rely on only our historical and examination skills to classify patients and optimally manage their disease? Further, how would limiting the types of rheumatologic testing that primary care providers could perform ultimately affect timely diagnoses and management of serious rheumatologic disease?

Unfortunately, while many may agree that optimizing testing in rheumatic diseases is an admirable goal, a major problem is the difficulty in designing, funding, and implementing robust studies to answer the questions presented above, and ultimately to assess the overall effect of testing on the longterm management and outcomes of rheumatic disease. Perhaps we will be limited to using clinical cohorts such as $\mathrm{CATCH}$, albeit with more substantial longterm data. Yet, despite these challenges, we should still strive to improve the use of tests in the management of rheumatic disease so that ultimately we are choosing wisely in the care of patients.

\section{KEVIN D. DEANE, $M D, P h D$}

Division of Rheumatology,

University of Colorado Denver School of Medicine, Aurora, Colorado, USA.

Address correspondence to Dr. K.D. Deane, University of Colorado Denver, Anschutz Medical Campus, Division of Rheumatology, Department of Medicine, 1775 Aurora Court, Mail Stop B-115, Aurora, Colorado 80045, USA.E-mail: Kevin.Deane@UCDenver.edu

\section{REFERENCES}

1. American Board of Internal Medicine. Choosing Wisely campaign. [Internet. Accessed October 21, 2015.] Available from: www.choosingwisely.org

2. American College of Rheumatology Choosing Wisely: Five things physicians and patients should question. [Internet. Accessed October 21, 2015.] Available from: www.choosingwisely.org/societies/ american-college-of-rheumatology/

3. Aletaha D, Neogi T, Silman AJ, Funovits J, Felson DT, Bingham CO 3rd, et al. 2010 Rheumatoid arthritis classification criteria: an American College of Rheumatology/European League Against Rheumatism collaborative initiative. Arthritis Rheum 2010; 62:2569-81.

4. Shu J, Bykerk VP, Boire G, Haraoui B, Hitchon C, Thorne JC, et al. Missing anticitrullinated protein antibody does not affect short-term outcomes in early inflammatory arthritis: from the Canadian Early Arthritis Cohort. J Rheumatol 2015;42:2023-8.

5. Whiting PF, Smidt N, Sterne JA, Harbord R, Burton A, Burke M, et al. Systematic review: accuracy of anti-citrullinated peptide antibodies for diagnosing rheumatoid arthritis. Ann Intern Med 2010;152:456-64; W155-66.

6. Lee DM, Schur PH. Clinical utility of the anti-CCP assay in patients with rheumatic diseases. Ann Rheum Dis 2003;62:870-4.

7. Lard LR, Visser H, Speyer I, vander Horst-Bruinsma IE, Zwinderman AH, Breedveld FC, et al. Early versus delayed treatment in patients with recent-onset rheumatoid arthritis: comparison of two cohorts who received different treatment strategies. Am J Med 2001;111:446-51.

8. Narvaez J, Díaz-Torné C, Ruiz JM, Hernandez MV, Torrente-Segarra V, Ros S, et al. Predictors of response to rituximab in patients with active rheumatoid arthritis and inadequate response to anti-TNF agents or traditional DMARDs. Clin Exp Rheumatol 2011;29:991-7.

J Rheumatol 2016;43:1-2; doi:10.3899/jrheum.151237

Personal non-commercial use only. The Journal of Rheumatology Copyright @ 2016 . All rights reserved. 